\title{
Growth of hydrodynamic perturbations in accretion disks: Possible route to non-magnetic turbulence
}

\section{Citation}

Mukhopadhyay, Banibrata, Niayesh Afshordi, and Ramesh Narayan. 2006. “Growth of Hydrodynamic Perturbations in Accretion Disks: Possible Route to Non-Magnetic Turbulence." Advances in Space Research 38 (12): 2877-79. https://doi.org/10.1016/j.asr.2005.09.048.

\section{Permanent link}

http://nrs.harvard.edu/urn-3:HUL.InstRepos:41384862

\section{Terms of Use}

This article was downloaded from Harvard University's DASH repository, and is made available under the terms and conditions applicable to Other Posted Material, as set forth at http:// nrs.harvard.edu/urn-3:HUL.InstRepos:dash.current.terms-of-use\#LAA

\section{Share Your Story}

The Harvard community has made this article openly available.

Please share how this access benefits you. Submit a story.

Accessibility 


\title{
Growth of Hydrodynamic Perturbations in Accretion Disks: Possible Route to Non-Magnetic Turbulence
}

\author{
Banibrata Mukhopadhyay, Niayesh Afshordi, Ramesh Narayan \\ Institute for Theory and Computation, Harvard-Smithsonian Center for \\ Astrophysics, 60 Garden Street, MS-51, Cambridge, MA 02138, USA
}

\begin{abstract}
We study the possible origin of hydrodynamic turbulence in cold accretion disks such as those in star-forming systems and quiescent cataclysmic variables. As these systems are expected to have neutral gas, the turbulent viscosity is likely to be hydrodynamic in origin, not magnetohydrodynamic. Therefore MRI will be sluggish or even absent in such disks. Although there are no exponentially growing eigenmodes in a hydrodynamic disk, because of the non-normal nature of the eigenmodes, a large transient growth in the energy is still possible, which may enable the system to switch to a turbulent state. For a Keplerian disk, we estimate that the energy will grow by a factor of 1000 for a Reynolds number close to a million.
\end{abstract}

Key words: accretion, accretion disk, hydrodynamic, turbulence, instabilities PACS: 97.10.Gz, 03.75.Kk, 47.27.J, 83.60.Wc

\section{INTRODUCTION}

In the context of Keplerian disks, while hydrodynamic simulations seem to confirm the absence of turbulence in the non-linear limit [1], the MagnetoRotational Instability [2], discovered by Balbus and Hawley [3] within ionized accretion flows, confirms the existence of MHD turbulence. This helps to understand the origin of shear stress or "viscosity". However the MRI driven instability fails to operate in disks with small ionization fraction. Examples of such systems are proto-planetary disks, outskirts of AGN accretion disks [4] etc.. As a result, the route to turbulence and subsequently accretion in neutral disks has remained one of the outstanding puzzles in modern astrophysics.

Laboratory experiments of Taylor-Couette systems (which are similar to accretion systems) seem to indicate that, the flow is unstable to turbulence for 
Reynolds numbers larger than a few thousand [5], even for subcritical systems. Based on this, Longaretti [6] concludes that the absence of turbulence in previous simulations [1] is due to their small effective Reynolds number.

The fundamental reason for subcritical turbulence may be the result of definite frequency modes not being orthogonal in a shear flow. Therefore, a suitably tuned linear combination of modes can show an arbitrarily large transient energy growth ${ }^{1}$. This transient growth may possibly lead to sustained hydrodynamic turbulence for large enough Reynolds numbers.

Here we analyze this transient growth phenomenon in the framework of accretion disks. We present the set of scaling relations for the transient growth as a function of the Reynolds number.

\section{BASIC EQUATIONS AND SCALING RELATIONS}

Let us define the shear frequency $2 A$ and the vorticity frequency $2 B$ as, $2 A=$ $-q \Omega, 2 B=(2-q) \Omega$. Then the momentum balance equations give

$$
\frac{d u}{d t}=2 \Omega v-\frac{\partial \hat{p}}{\partial x}+\nu \nabla^{2} u, \frac{d v}{d t}=-2 B u-\frac{\partial \hat{p}}{\partial y}+\nu \nabla^{2} v, \frac{d w}{d t}=-\frac{\partial \hat{p}}{\partial z}+\nu \nabla^{2} w
$$

along with the incompressibility relation $\partial u / \partial x+\partial v / \partial y+\partial w / \partial z=0$, where $\hat{p}=P / \rho$. Symbols $u, v, w$ and $\Omega$ are three velocity components and angular frequency respectively, $\nu, P, \rho$ are dynamical viscosity, pressure and density of the system respectively and $q$ is a parameter which controls the flow pattern (whether a Keplerian disk, a constant angular momentum disk or plane Couette flow). The Lagrangian time derivative $d / d t$ is, $d / d t=\partial / \partial t-q \Omega x \partial / \partial y$. Here $x$ varies from $-L$ to $+L$, and $y$ and $z$ from 0 to $2 \pi / k_{y}$ and $2 \pi / k_{z}$ respectively, where $k_{x}, k_{y}, k_{z}$, are the corresponding components of wave-vectors. The boundary conditions are $u=v=w=0$ at $x= \pm L$.

\subsection{Constant Angular Momentum Disks and Plane Couette Flow}

It is clear from (1) that the set of equations is very symmetrical for a constant angular momentum $(q=2)$ and plane Couette $(\Omega=0)$ flow, except that $x$ and $y$ are interchanged. Therefore the growth is identical in the two cases. As the previous analytical/numerical studies (e.g. [8,7]) showed that the growth

1 Growth is defined as the ratio of energy at a particular instant to that at the beginning. 
is maximum for vertical perturbations $\left(k_{y}=0\right)$, here we consider such perturbation. Noting that the Reynolds number is, $R=|2 A| L^{2} / \nu$, we find that the maximum growth in energy for a given $k_{z}$ is

$$
G_{\max }\left(k_{z}, R\right)=\left(\frac{u^{2}(t)+v^{2}(t)+w^{2}(t)}{u^{2}(0)+v^{2}(0)+w^{2}(0)}\right)_{\text {optimum }}=\frac{R^{2} k_{z}^{2} L^{2} e^{-2}}{\left[\frac{1}{2} \pi^{2}+k_{z}^{2} L^{2}\right]^{3}}
$$

Maximizing this over $k_{z}$, we find that the optimum wavevector is $k_{z} L=\pi / 2=$ 1.57. The maximum growth factor and the corresponding time are

$$
G_{\max }(R)=0.82 \times 10^{-3} R^{2},|2 A| t_{\max }(R)=0.13 R .
$$

Clearly the growth scales as $R^{2}$ and therefore can be very large even for modest $R$. For example, when $R=1200$, the above relations give $G_{\max }(R)>1000$, which is probably large enough to induce non-linear feedback and turbulence.

\subsection{Keplerian Disk}

Here we study eqns. (1) for $q=1.5$. As the earlier analysis [7] hinted that $k_{z}=0$ generates the best growth, we consider such perturbations.

We consider a plane wave that is frozen into the fluid and is sheared along with the background flow. If the flow starts at time $t=0$ with initial wave-vector $\left\{k_{x i}, k_{y}\right\}$, the $k_{x}$ at later time is given by $k_{x}(t)=k_{x i}+q \Omega k_{y} t$. The maximum energy growth is then approximately

$$
G_{\text {max }}\left(k_{x i}, k_{y}, R\right) \sim \frac{k_{x i}^{2} L^{2}}{(1.7)^{2}+k_{y}^{2} L^{2}} \exp \left(-\frac{2}{3 R} \frac{k_{x i}^{3} L^{2}}{k_{y}}\right) .
$$

when $k_{x, \min } L=1.7$. Maximizing this with respect to $k_{x i}$ and $k_{y}$, we obtain

$$
G_{\max }(R) \sim 0.13 R^{2 / 3},|2 A| t_{\max }(R) \sim 0.88 R^{1 / 3}
$$

Also maximizing with respect to $k_{x i}$, keeping $k_{y}$ and $R$ fixed, we obtain

$G_{\text {max }}\left(k_{y}, R\right)=\frac{\left(k_{y} L\right)^{2 / 3}}{(1.7)^{2}+\left(k_{y} L\right)^{2}} e^{-2 / 3} R^{2 / 3},|2 A| t_{\text {max }}\left(k_{y}, R\right)=\left(k_{y} L\right)^{-2 / 3} R^{1 / 3}$

We see that the maximum growth scales as $R^{2 / 3}$. Though this rate of increase is less than that for a $q=2$ disk and plane Couette flow, still, at a large enough 
$R$, the growth can become large, and may cause turbulence in a Keplerian disk. For example, for $R=10^{6}, G_{\max }(R)>1000$, which by analogy with a $q=2$ disk may be enough to cause turbulence.

\section{CONCLUSION}

We have shown that significant transient growth of perturbations is possible in a Keplerian flow. Although the system does not have any unstable eigenmodes, because of the non-normal nature of the eigenmodes a significant level of transient energy growth is possible at a large Reynolds number for appropriate choice of initial conditions. We argue that a plausible critical Reynolds number for sustaining hydrodynamic turbulence in a Keplerian disk is $\sim 10^{4}-10^{6}$ (for detailed discussions, see [7]). We base this on the expectation that once the growth crosses the threshold, secondary instabilities of various kinds, such as the elliptical instability (see e.g. [9]), might serve as a possible route to selfsustained turbulence. However, it remains to be verified that these instabilities are present. Also, even if they are present, one will need to investigate whether they lead to non-linear feedback and 3-dimensional turbulence.

This work was supported in part by NASA grant NAG5-10780 and NSF grant AST 0307433.

\section{References}

[1] S. Balbus, J. Hawley \& J. Stone, ApJ 467, 76 (1996); J. Hawley, S. Balbus, W. Winters, ApJ 518, 394 (1999)

[2] S. Chandrasekhar, Proc. Nat. Acad. Sci. 46, 53 (1960)

[3] S. Balbus \& J. Hawley, ApJ 376, 214 (1991)

[4] C. Gammie \& K. Menou, ApJ 492, L75 (1998)

[5] D. Richard, Ph.D. thesis, Univ. Paris VII (2001)

[6] P. Longaretti, ApJ 576, 587 (2002)

[7] B. Mukhopadhyay, N. Afshordi \& R. Narayan, ApJ, to appear (2005), astro-ph/0412193 to appear in Proc. of 22nd Texas Symposium on Relativistic Astrophysics at Stanford University, Stanford, California, Dec 13-17, 2004, astro-ph/0501468 N. Afshordi, B. Mukhopadhyay \& R. Narayan, ApJ, to appear (2005), astro-ph/0412194

[8] K. Butler \& B. Farrell, Phys. Fluids A 4, 1637 (1992)

[9] R. Kerswell, Ann. Rev. Fluid Mech. 34, 83 (2002) 